\title{
Epidemiology of Invasive Candidiasis and Challenges for the Mycology Laboratory: Specificities of Candida glabrata
}

\author{
Paula Sampaio • Célia Pais
}

Published online: 21 June 2014

(C) Springer International Publishing AG 2014

\begin{abstract}
Candida species are an increasing cause of invasive candidiasis (IC), resulting in significant mortality and morbidity. Although Candida albicans remains the predominant etiologic agent of invasive fungal infections in hospital settings, the incidence of new infections from non-albicans Candida species is steadily increasing, and important geographical differences in species distribution and patterns of in vitro antifungal susceptibilities have been reported. In this article, we review the recent epidemiology of Candida invasive infections, and discuss trends in incidence, mortality, species distribution, and antifungal resistance, emphasizing the particular situation of $C$. glabrata. We also address the techniques available for diagnosis of IC, the classic culture techniques and the non-culture based methods, including the recent recommendation of the European Fungal Infection Study Group (EFISG) of the European Society of Clinical Microbiology and Infectious Diseases (ESCMID) about different techniques and procedures to detect IC, considering specificities with $C$. glabrata detection.
\end{abstract}

Keywords Epidemiology · Invasive candidiasis · Candidaemia $\cdot$ Candida glabrata $\cdot$ Candida species . Antifungal susceptibility $\cdot$ Diagnosis techniques $\cdot$ European Fungal Infection Study Group remommendations

\section{Introduction}

Fungi have emerged as a major cause of human infection, with yeasts belonging to the genus Candida being the major

P. Sampaio $(\bowtie) \cdot$ C. Pais

Centre of Molecular and Environmental Biology (CBMA),

Department of Biology, University of Minho, Campus de Gualtar,

4710-057 Braga, Portugal

e-mail: psampaio@bio.uminho.pt opportunistic fungal pathogens. Before the 1970s, fungal infections were easily curable and mainly superficial; however, with the later increase in number of immunocompromised patients, the number of serious life-threatening fungal infections increased significantly. Several factors have contributed to this increase, but paradoxically, medical progress and the use of new therapies developed to provide a longer and better life has had a significant impact on the increase of the number of patients susceptible to fungal infections [1]. Numerous risk factors have been associated with serious life-threatening Candida infections, candidemia, and invasive candidiasis (IC), including exposure to broad-spectrum antimicrobial agents, cancer chemotherapy, neutropenia, prior surgery (especially gastrointestinal), indwelling vascular catheter, total parenteral nutrition, and extremes of age.

Although the list of opportunistic fungi causing invasive infections has been increasing every year, Candida species remains the most important cause of opportunistic mycoses worldwide. Nowadays, approximately half of the cases of candidoses are caused by non-albicans species, C. glabrata, C. parapsilosis, C. tropicalis, C. krusei, C. guilliermondii, and C. dubliniensis. These species are emerging as both colonizers and pathogens able to cause superficial and systemic infections [2]. Some of these species, such as C. glabrata and C. krusei, are more resistant to antifungal agents [3], while others, like C. dubliniensis, rapidly develop fluconazole resistance during clinical therapy [4]. Regardless of the growing understanding of the epidemiology of opportunistic fungal infections, the ability to prevent and treat Candida infections still has serious deficiencies $[5,6]$.

In this article, we review the recent epidemiology of Candida invasive infections, and discuss trends in incidence, mortality, species distribution, and antifungal resistance, emphasizing the particular situation of $C$. glabrata. We also address the techniques available for diagnosis of IC, the classic culture techniques and the non-culture based methods, 
including the latest recommendation of the European Fungal Infection Study Group (EFISG) of the European Society of Clinical Microbiology and Infectious Diseases (ESCMID) about different techniques and procedures to detect IC, considering specificities with $C$. glabrata detection.

\section{Epidemiology and Mortality Rate of Invasive Candidiasis}

Invasive candidiasis can be defined as a deep-seated, frequently multi-organ infection, including candidemia (the presence of any species of the genus Candida in the blood). IC is an important cause of morbidity and mortality associated with increased hospital stays and high healthcare costs. Although C. albicans is the most common cause of invasive fungal infections in hospital settings, the incidence of new infections from non-albicans Candida species is steadily increasing, and important geographical differences in species distribution and patterns of in vitro antifungal susceptibilities have been reported $[7,8 \bullet]$. Population-based studies indicate that the incidence of disseminated candidiasis varies within different geographic areas. In the USA these values ranged from 0.28 to 0.96 episodes/1,000 admissions [9], while in Europe the incidence values varied from 0.1 to 0.53 episodes $/ 1,000$ admissions [1, 7, 10-13]. In Latin America the values ranged from 0.33 to 1.96 episodes/1,000 admissions [14], with Colombia presenting the higher incidence value. Numerous factors may contribute to these differences, and differences in the design of the population studies may be crucial, including the type of patients studied (neonates, children, or adults), the underlying disease, and medical practices, especially the use of long-term vascular catheters and antibacterial and/or antifungal usage patterns $[15,16]$. Surveillance studies have reported that during the last decade, although the number of immunocompromised patients continues to grow, the mortality associated with IC remained relatively steady, at approximately 0.4 deaths per 100,000 population [1]. The reasons for this may be the introduction of new antifungal agents that are more effective and less toxic [17, 18], as well as the changes in immunosuppressive regimens that are less aggressive to patients. Nevertheless, neither the incidence nor the mortality associated with IC is declining, warranting continued investigation and understanding of the challenge that IC presents to healthcare systems.

\section{Species Distribution of the Etiological Agents of Invasive Candidiasis}

More than 20 different species of Candida have been associated as etiological agents of IC, and the list of reported species continues to grow as laboratories are encouraged to provide an identification to the species level to guide antifungal therapy
[19]. Despite the predominance of $C$. albicans worldwide, a decreasing trend in the isolation of this species is evident over time [19]. In contrast, the rates of isolation of C. glabrata, C. tropicalis, and C. parapsilosis are steadily increasing. Curiously, in the last decade, the rates of isolation of other Candida species such as C. krusei, C. guilliermondii, C. lusitaniae, C. kefyr, C. rugosa, and C. famata did not vary significantly [19]. All these species are emerging as both colonizers and pathogens able to cause superficial and systemic infections [20].

Comparative studies on species distribution are always difficult to interpret due to temporal and geographic variations as well as to differences in the characteristics of the studied hospital. However, C. glabrata has undoubtedly emerged as an important opportunistic fungal pathogen in the USA [3, 19, 21]. Virtually all US-based surveys show $C$. glabrata to rank second to C. albicans as a cause of IC. In contrast, in Europe, although C. glabrata has also increased as a cause of IC and may rank second after $C$. albicans in some studies, it has not increased to the extent that it has in the USA, despite an increase in the use of fluconazole [11, 22-29]. However, most notable is the comparatively low frequency of $C$. glabrata as a cause of IC in Latin America and Mediterranean countries, in which C. parapsilosis is, after C. albicans, often the most frequently recovered etiological agent of IC [15, 20, 30]. Figure 1a shows the percentage of $C$. glabrata isolation in the countries were population-based studies are available.

The reasons for such dramatic variation in the frequency of C. glabrata as a cause of IC are unclear but may include exposure to azoles, patient age, underlying disease, geographic location, or other unknown factors [1]. It is well known that C. glabrata rapidly acquires fluconazole resistance with azole exposure and can up-regulate drug efflux pump genes with as little as 4 days of in vitro fluconazole exposure [31], which is generally associated with the increased incidence. Indeed, it has been documented that a change in the hospital's antifungal prophylactic strategy correlates with the risk of $C$. glabrata IC. A reduction in fluconazole treatment has been shown to result in a decrease in the incidence of fungemia due to $C$. nonalbicans, including C. glabrata [32], and an increase in fluconazole prophylaxis correlates with a higher risk of C. glabrata IC [15]. However, recent studies did not find a significant association between prior exposure to fluconazole and higher risk of subsequent $C$. glabrata IC $[2,33]$. Instead, a significant trend toward an increased proportion of IC due to C. glabrata and older patients was documented [34]. Age above 60 years not only increases risk of fungemia but also the risk of dying from the infection (Fig. 1b). In the latter studies, the most common predisposing factors for C. glabrata IC were the use of broad-spectrum antibiotics, use of central venous catheter, stay in an intensive care unit (ICU), renal failure, and parenteral nutrition [34]. Taken together, these findings indicate that prior exposure to fluconazole is not the 
Fig. 1 Geographic variation of Candida glabrata isolated from invasive candidiasis and distribution of the most frequent Candida species according to patient age. a Geographic distribution of the percentage of isolation of C. glabrata. b Distribution of the percentage of bloodstream isolates of

C. albicans, C. glabrata, and C. parapsilosis found in each patient age group; these data were collected from population-based studies [11, 27, 53]

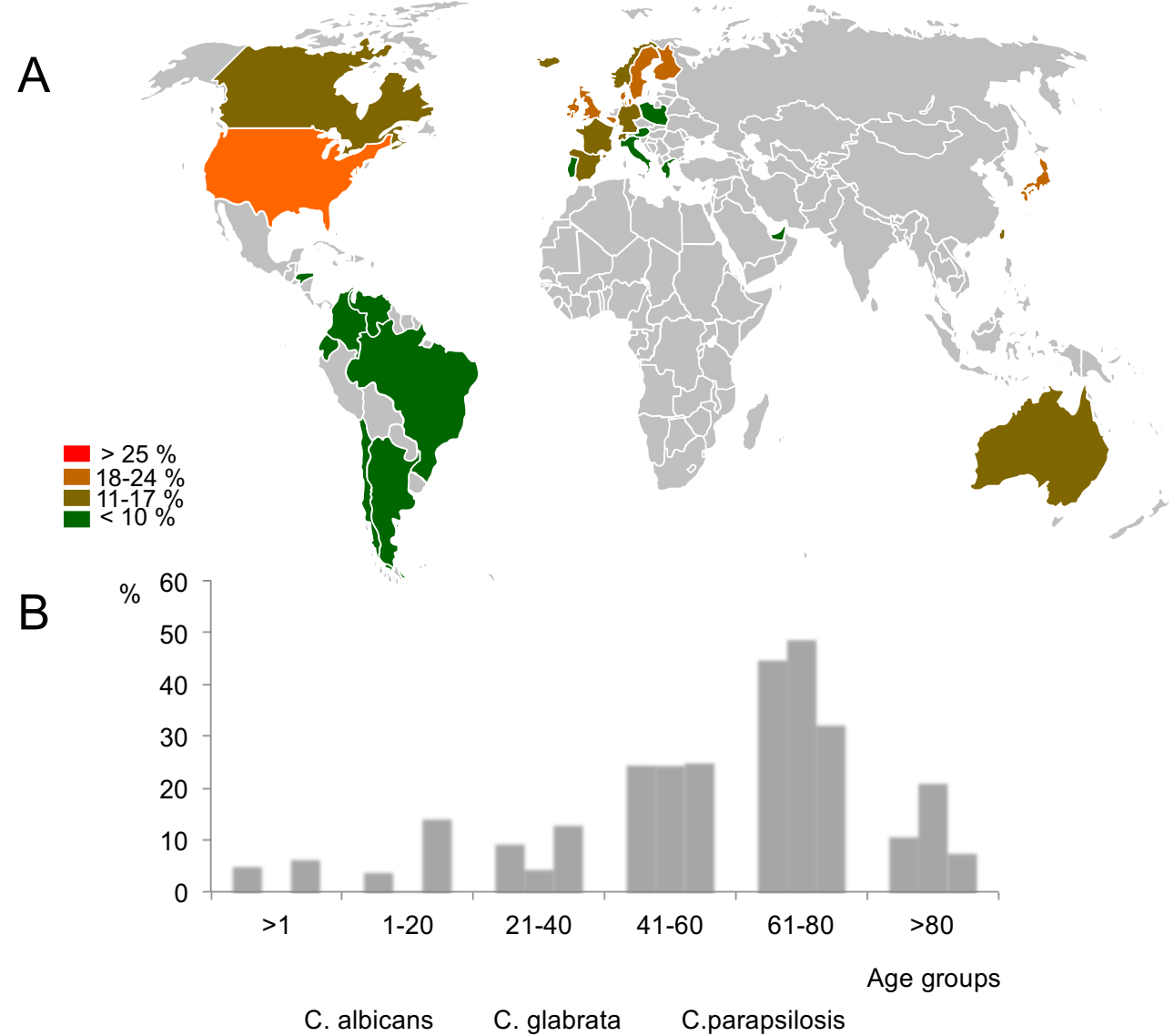

single and most important predisposing factor for subsequent C. glabrata IC. Patient age, exposure to specific antibacterial agents, and severity of underlying disease are also important risk factors promoting C. glabrata candidemia.

\section{Antifungal Susceptibility of Isolates from Invasive Candidiasis}

It is now recognized that early and appropriate antifungal therapy has a positive effect on the outcome of patients with IC, as well as lowering hospital resource utilization [35-37]. Thus, in vitro antifungal susceptibility testing plays an important role in therapeutic antifungal drug selection and is also important in epidemiologic studies to track the development of antifungal resistance [38, 39].

One of the most desirable properties of an antifungal drug is its ability to kill the fungus in order to allow a more rapid and complete organ clearance during the infection. Among the antifungal agents currently in use in clinical practice, the echinocandins and amphotericin B (AMB) are considered to exert a fungicidal activity $[18,40]$. Detection of resistance to AMB has been problematic due to the very narrow range of minimal inhibitory concentrations (MICs) obtained by the Clinical and Laboratory Standards Institute (CLSI) broth dilution procedure and standardization of other proposed methods [41, 42]. Several reports indicate that the E-test provides better discrimination between AMB-susceptible and AMB-resistant Candida isolates than does the CLSI reference method and they suggest that MICs obtained by this test are more predictive of treatment outcome [5, 7, 43, 44]. Antifungal surveillance programs have shown that C. glabrata and C. krusei exhibit decreased susceptibility to AMB $(113,120,202,215,216,323)$ as well as a delayed drug-killing kinetics compared with $C$. albicans $[6,17,45]$. Several studies indicate that overall resistance to AMB is uncommon [46]; however, higher MICs were observed in sequential isolates of C. glabrata recovered from critically ill patients [47].

The triazole antifungal agents are the most frequently used in the treatment of IC. The broad use of fluconazole since its introduction has raised concerns regarding the emergence of azole resistance $[5,7,9,14,19,27,48-50]$. The detection and tracking of azole resistance trends has been facilitated by the availability of a standardized broth microdilution method [38, 51]. Although fluconazole resistance was detected in only a small proportion of C. albicans ( $0.1 \%)$, C. tropicalis (3.2\%), and $C$. parapsilosis $(5.0 \%)$ isolates, these species were observed to account for about $34 \%$ of fluconazole-resistant isolates [8•]. Several studies have confirmed that, over the last 
decade, fluconazole resistance among Candida isolates has remained infrequent worldwide, except for isolates of C. glabrata $[3,15,19,52,53,54 \cdot, 55]$. One of the largest and representative studies, which tested more than 200,000 strains of Candida spp. isolated between June 1997 and December 2005 from 134 different medical centers worldwide, showed that approximately $90 \%$ of all Candida spp. isolates were susceptible to fluconazole, but only $68.9 \%$ of all C. glabrata were susceptible [19]. The resistance rates for fluconazole among isolates of $C$. glabrata varied considerably among the various geographic regions, with the lowest rates seen in the Asia-Pacific region and the highest in North America [19]. Thus, given the prominence of C. glabrata as a cause of IC in all geographic regions, its progressive worldwide acquisition of fluconazole resistance, the well documented cross-resistance between fluconazole and other triazoles (voriconazole, posaconazole, and itraconazole), and the susceptibility of this species to azoles in the context of prior exposure, continuous antifungal susceptibility testing and monitoring of $C$. glabrata isolates is of extreme importance and may result in lower overall treatment costs for these patients [39].

The echinocandin class of antifungal agents acts by inhibiting the synthesis of the yeast cell wall $1,3-\beta$-D-glucan and is recommended for the treatment of IC due to its excellent clinical efficacy coupled with low toxicity [18, 56, 57]. Since the introduction of fluconazole for the treatment of IC, empirical antifungal therapy has been driven by fear of decreased susceptibility and cross-resistance to other azoles of C. glabrata $[58,59]$. Thus, echinocandins were recommended as first-line therapy for the treatment of IC in patients with prior exposure to azoles and/or infection with C. glabrata because these new antifungals have distinct mechanisms of action and cross-resistance between the two classes is unknown [60]. Caspofungin was the first to be approved for the treatment of IC and has been the more extensively used over past years, raising concerns about the emergence of resistance. So far, clinical experience with caspofungin in IC has been good, with several surveys showing that resistance to one or more echinocandins is relatively low [17, 61, 62]. Data from global surveys demonstrate that the frequency of echinocandin resistance among clinical isolates of C. glabrata ranges from 1 to $3 \%$ and is higher among isolates from North America (3\%) than among those from Europe (1\%), Latin America (0.0\%), or the Asia-Pacific region $(0.0 \%)[8 \cdot, 25,61,63]$. Additionally, it has been reported that C. glabrata has the ability to mutate in vivo following prolonged exposure to antifungal agents, acquiring sequential resistance to the drugs (known as multidrug resistance [MDR]) [64]. MDR, resistance to two or more classes of antifungal agents, is a serious complication during treatment of opportunistic fungal infections and, in past years, several reports from medical centers provide indication of MDR strains of $C$. glabrata [47, 64, 65]. These MDR C. glabrata strains presented FKS mutations, which are known to be responsible for the caspofungin-resistance phenotype [61]. The documentation of FKS mutations in isolates of C. glabrata showing in vitro resistance to both azoles and echinocandins suggests the sequential accumulation of acquired resistance mechanisms that, due to the haploid nature of the species, are dominantly expressed $[54 \bullet, 66]$.

\section{Diagnosis of Invasive Candidiasis}

The clinical syndromes of invasive fungal infections present different degrees of severity, and are not very different from those caused by bacteria. To diagnose, that is to establish the presence or absence of a disease, clinicians use tests, often as a package or strategy, including signs and symptoms, imaging, and biochemistry. The choice of a diagnostic test is based on evidence regarding accuracy (sensitivity and specificity), that is, how well the test classifies patients correctly as having or not the disease. The underlying assumption is that obtaining a better idea of whether a target condition is present or absent will result in improved outcome [67]. In the case of IC, it is widely recognized that the timing of antifungal therapy has a major impact on the mortality, thus, prompt diagnosis is extremely important so as to initiate antifungal therapy as early as possible and to select the most appropriate antifungal drug [35-37]. In addition to diagnostic tools, understanding of the local epidemiology, patient risk factors, and resistance profiles of Candida species is essential. The major drawbacks in the utilization of diagnostic tools are the lack of standardization and grading of the available methods. Recently, efforts have been made to grade accuracy of the procedures, quality of evidence, and strength of the recommendation for diagnostic tests $[67,68 \bullet]$.

Invasive candidiasis frequently involves multi-organ infections and candidemia. Although blood cultures are negative in about one-third of ICs [23], they are essential for the diagnosis of candidemia. Several international guidelines regarding general recommendations on how to take and process blood samples, including the frequency of takes and the incubation period, are available to ensure the optimal isolation of microorganisms $[68 \cdot, 69-71]$. When these recommendations are followed, the sensitivity of this technique is around 50 $70 \%$. Reports suggest that the choice of blood culture system may influence the recovery of $C$. glabrata, and that the BACTEC $^{\text {TM }}$ system may be inferior to the BacT/ALERT ${ }^{\circledR}$ system in this respect [53]. Thus, the recommendation of the EFISG/ESCMID panel was that blood culture is an essential investigation, meaning that it must be done whenever possible, and they recommend the use of automated validated blood culture systems (Table 1) [68•]. Once a positive culture is obtained, the identification to species level is mandatory, 
Table 1 Summary of the recommendations and level of evidence assigned by the EFISG/ESCMID panel for the diagnostic tests used for invasive candidiasis and candidemia. Adapted from Cuenca-Estrella et al. [68•]

\begin{tabular}{|c|c|c|c|c|}
\hline Specimen & Test & Recommendation & Level of evidence ${ }^{\mathrm{a}}$ & Specificities for Candida glabrata detection ${ }^{\mathrm{b}}$ \\
\hline Blood & Blood culture & Essential investigation & NA & BacT/ALERT system is better for $C$. glabata isolation \\
\hline \multirow[t]{4}{*}{ Tissue } & Direct microscopy and histopathology & Essential investigation & NA & C. glabrata does not filament \\
\hline & Culture & Essential investigation & NA & - \\
\hline & Immuno-histochemistry & No recommendation & No data & - \\
\hline & Tissue PCR & No recommendation & No data & - \\
\hline \multirow[t]{4}{*}{ Serum } & Mannan/anti-mannan & $\begin{array}{l}\text { No recommendation } \\
\text { Recommended }^{\mathrm{c}}\end{array}$ & $\begin{array}{l}\text { No data } \\
\text { II }\end{array}$ & - \\
\hline & $\beta$-1,3-D-glucan & Recommended & II & Pre-treatment with echinocandins reduces accuracy \\
\hline & SeptiFast system & No recommendation & No data & - \\
\hline & In-house PCR & No recommendation & No data & - \\
\hline
\end{tabular}

$I C$ invasive candidiasis, $N A$ not applicable, $P C R$ polymerase chain reaction

${ }^{a}$ II indicates evidence from (1) at least one well designed prospective single-center cross-sectional or cohort study, or (2) a properly designed retrospective multicenter cross-sectional or cohort study, or (3) from case-control studies

b _ indicates no specificity

${ }^{c}$ Based on the lack of studies for IC

${ }^{d}$ Recommendation exclusively for candidemia

because antifungal therapy can vary according to Candida species.

Classical diagnostic methods such as direct microscopy, histopathology, and culture present a limited sensitivity to detect IC, and their usefulness depends on the possibility of obtaining deep tissue samples, which in many cases is not possible due to the patient's condition. However, when possible, a tissue sample should be taken, since knowing the infective species is essential for a correct therapeutic approach. The tissue samples collected aseptically should be processed promptly and used for both histopathology (rapidly placed in fixative) and culture (not placed in histopathology fixatives) if possible. Microscopic visualization of fungal elements in fixed tissue, after periodic acid-Schiff (PAS) or silver staining, is the basis for the diagnosis of IC. Candida spp. are yeasts that can produce pseudohyphae. Thus, this structure may help in differentiation from other yeasts and molds that produce only true hyphae in tissue; however, C. glabrata does not produce pseudohyphae. Microscopic examination of fixed tissues requires expertise, for interpretation and morphology cannot be used for definitive identification [72].

To avoid multiplication of organisms, samples intended for culture should be processed as soon as possible and inoculated in fungal-selective agar media such as Mycosel (BBL) and Mycobiotic (Difco) for 5-14 days. Yeast isolation from normally sterile tissues or fluids is usually indicative of deepseated infection, but negative cultures do not exclude Candida infection [68•]. Identification of the isolate to species level is always necessary. Although the application of these techniques has shown positive results in some studies, very few clinically validated immunohistochemistry commercial kits are available: only the species-specific Rabbit anti C. albicans, type A:Biotin (Serotec) for detection of fungal infections. The EFISG/ESCMID panel recommends that direct microscopy and histopathology is an essential investigation, and must be carried out if possible (Table 1).

Alternative diagnostic procedures, also known as nonculture-based methods, are increasingly used in the management of patients at risk of IC to improve and anticipate detection of candidemia. These methods are based on the detection and quantification of fungal biomarkers and metabolites from serum of patients. The major serological tests for Candida infections include measurement of serum mannan (Mn) and anti-Mn antibodies or $\beta$-1,3-D-glucan.

Mannan is a component of the Candida cell wall, accounting for up to $7 \%$ of the cell wall dry weight, and is one of the main Candida Ags that circulate in blood during infection [73]. Initial observations showing that mannanemia was preferentially observed in the absence of anti-Mn antibodies and that, vice versa, high levels of anti-Mn antibodies were generally not associated with mannanemia led to the idea that the combined detection of mannanemia and anti-Mn antibodies would be a useful diagnostic procedure [74]. Enzyme-linked immunosorbent assays (ELISAs) quantifying Mn and anti-Mn antibodies are marketed as Platelia Candida Antigen Plus and Platelia Candida Antibody Plus (Bio-Rad Laboratories). Several studies have confirmed the high efficacy of the diagnosis of candidemia, with sensitivities around $80 \%$ and specificity of $85 \%$ [71]. The sensitivity of both $\mathrm{Mn}$ and anti-Mn varied for different Candida species, and was the highest for C. albicans, followed by C. glabrata and C. tropicalis [75]. 
This method can help detect candidemia earlier than can blood cultures and shows a high negative predictive value (>85\%), useful in the ruling out of infection. Thus, the EFISG/ESCMID group considered this method to be recommended for the diagnosis of candidemia, meaning that this technique is accurate in $50-70 \%$ of cases, and suggested its incorporation in a diagnosis strategy [68•]. However, very few studies are available regarding detection of IC by quantification of $\mathrm{Mn} / \mathrm{anti}$ $\mathrm{Mn}$; thus, the EFISG/ESCMID panel could not recommend this test for detection of IC (Table 1).

The $\beta$-1,3-D-glucan is also a component of the cell wall of most fungi except Zygomycetes and Cryptococcus, and constitutes approximately $40 \%$ of the Candida cell wall dry weight [73]. The $\beta-1,3-\mathrm{D}$-glucan detection is thus considered a panfungal diagnostic method and was included in the European Organization for Research and Treatment of Cancer/Mycosis Study Group (EORTC/MSG) diagnostic criteria for invasive fungal infections in 2008, for all types of patients [76]. Several assays are commercially available for diagnostic use: Fungitell (Associates of Cape Cod, Inc., East Falmouth, MA, USA), Fungitec-G (Seikagaku, Kogyo, Tokyo, Japan), Wako (Wako Pure Chemical Industries, Ltd., Tokyo, Japan), and Maruha (Maruha-Nichiro, Foods Inc., Tokyo, Japan). The kits differ in the source of the substrate used for the chromogenic reaction, that is, the amoebocyte lysate from the horseshoe crab, Limulus polyphemus (USA) or Tachypleus tridentatus (Japan). The Fungitell assay has been approved by the US FDA and is available in the USA and Europe, and the other tests are available in Japan. Several studies have been performed to assess the sensibility and specificity of this method using data from cross-sectional, cohort, and case-control studies on the diagnosis of candidemia. The sensitivity of glucan detection was $>65 \%$ in most studies, with a cut-off value of $80 \mathrm{pg} / \mathrm{mL}$, with specificity rates $>80 \%[68 \bullet, 71]$. $\beta-1,3-\mathrm{D}$-glucan assay was revealed to be of great utility in adult patients without hematological diseases, such as surgical or medical ICU patients; a single positive assay at the onset of sepsis was found to help discriminate patients at high risk of invasive fungal infection, with a negative predictive value of nearly $99 \%$ [77].

The non-culture-based serological methods present several common limitations, of which the most important is the lack of ability to discriminate between infection and colonization. In fact, some patients who were positive for IC by ELISA had high levels of Candida colonization (Candida was present in central line tips, wound swabs, non-directed bronchial lavage fluid, urine, and skin swabs) [78]. Additionally, it is known that inhibition of the enzymes responsible for the cell wall $1,3-\beta$-D-glucan by echinocandins reduces the amount of the component in the cell wall [79], which poses an additional challenge regarding $\beta$-1,3-D-glucan test, particularly problematic in IC infections due to C. glabrata. In this view, attention should be paid to the potential causes of false- negative results (due to antifungal prophylaxis or therapy [particularly echinocandins], or Zygomycetes and Cryptococcus infections) and false-positive results (due to blood transfusions or blood-derived products, hemodialysis/ hemofiltration, beta-lactam antibiotics, immunoglobulins (Igs), cellulose dressings, contamination of specimens at the bedside or during pre- or analytical processing by organic dusts or wastes, and bacterial infections) [71, 80]. The EFISG/ESCMID group considered this method as recommended for the diagnosis of candidemia and suggested its incorporation in diagnosis strategy (Table 1) [68•].

Other non-culture-based methods include the molecular typing techniques, based on polymerase chain reaction (PCR). The use of PCR in the detection of systemic fungal infection has been extensively published and provides potential in terms of sensitivity and specificity. Studies comparing PCR-based techniques with serological assays demonstrated that patients became PCR positive a mean of 2-4 days prior to becoming ELISA positive [78].

A range of PCR targets have been used, but the most frequently used, due to their universal nature and large copy number, are the ribosomal RNA (rRNA) genes (18S, 28S, and 5.8S rRNA genes). Their polymorphism has mainly been analyzed through post-amplification methods such as restriction fragment length polymorphism (RFLP), nested PCR, single-strand confirmation polymorphism, hybridization with specific probes or sequencing, all of which enhance specificity in the identification of the genus or species causing infection [81]. A systematic review assessing the diagnostic accuracy of PCR-based methods to detect Candida spp. directly in blood samples confirmed their high sensitivity and specificity, both above $90 \%$, for the diagnosis of IC [81]. In the study, all PCRbased methods used for the identification of Candida spp. to the genus or species level, including standard, nested, realtime, or reverse transcriptase PCR, using single or multiplex assays, as well as any PCR target that had been previously published, were evaluated. A higher sensitivity was observed with whole-blood (rather than serum) samples, the use of the QIAamp kit for DNA extraction, the use of rRNA or P450 genes as the target for PCR. However, for diagnostic purposes, the most promising PCR techniques are the automatic ones that use fluorescently labeled specific probes and real-time PCR. These techniques provide a species/genus level of identification and remove the need for post-amplification handling and the potential for contamination. The LightCycler SeptiFast system (Roche) is a real-time PCR-based commercial kit to detect bacteria and fungi (C. albicans, C. tropicalis, C. parapsilosis, C. krusei, C. glabrata, and Aspergillus fumigatus) in blood samples. With the advances in PCRbased techniques, previously unrecognized 'cryptic' species have been identified and added to the list of human pathogens. Recently, two other pathogenic species, $C$. nivariensis and C. bracarensis, have been described as being closely related 
to $C$. glabrata $[82,83]$. The true clinical relevance of possible misidentifications are under evaluation, since both species are rare, at around $0.2 \%$ prevalence [84]. However, in vitro susceptibility studies of $C$. bracarensis isolates were comparable to those found with $C$. glabrata, thus, it would be prudent to continue to monitor for these emerging pathogens. Although some studies have reported cases of candidemia being detected by the LightCycler SeptiFast system, the number of studies and cases are still limited, and it has been very difficult to delineate recommendations. Thus, due to lack of data, no recommendation was made by the EFISG/ESCMID group regarding the SeptiFast PCR kit or other in-house PCRbased methods for the diagnosis of IC (Table 1) [68•].

\section{Conclusions}

Invasive candidiasis is an important cause of morbidity and mortality and is associated with increased hospital stays and high healthcare costs. IC constitutes a challenging diagnostic and therapeutic problem. Recently, the EFISG of the ESCMID evaluated the accuracy, quality of evidence, and strength of recommendation for several diagnostic tests, being now possible to recommend the best for IC and candidemia. Recent epidemiological studies on Candida species associated with IC have confirmed the trend for both species distribution and antifungal resistance patterns to vary across geographic regions. MDR C. glabrata isolates are more frequently observed and constitute a serious problem, particularly for older patients with prior exposure to azole treatment. Thus, clinical awareness, knowledge of local epidemiology, and antifungal resistance are important factors for an early diagnosis and effective treatment of IC, particularly that due to $C$. glabrata.

\section{Compliance with Ethics Guidelines}

Conflict of Interest Paula Sampaio and Célia Pais, declare no conflicts of interest.

Human and Animal Rights and Informed Consent This article does not contain any studies with human or animal subjects performed by any of the authors.

\section{References}

Papers of particular interest, published recently, have been highlighted as:

- Of importance

1. Pfaller MA, Diekema DJ. Epidemiology of invasive candidiasis: a persistent public health problem. Clin Microbiol Rev. 2007;20(1): 133-63.
2. Chen $\mathrm{SC}$ et al. Candidaemia with uncommon Candida species: predisposing factors, outcome, antifungal susceptibility, and implications for management. Clin Microbiol Infect. 2009;15(7):662-9.

3. Diekema D et al. The changing epidemiology of healthcareassociated candidemia over three decades. Diagn Microbiol Infect Dis. 2012;73(1):45-8.

4. Moran GP et al. Antifungal drug susceptibilities of oral Candida dubliniensis isolates from human immunodeficiency virus (HIV)infected and non-HIV-infected subjects and generation of stable fluconazole-resistant derivatives in vitro. Antimicrob Agents Chemother. 1997;41(3):617-23.

5. Hajjeh RA et al. Incidence of bloodstream infections due to Candida species and in vitro susceptibilities of isolates collected from 1998 to 2000 in a population-based active surveillance program. J Clin Microbiol. 2004;42(4):1519-27.

6. Pappas PG et al. Guidelines for treatment of candidiasis. Clin Infect Dis. 2004;38(2):161-89.

7. Tortorano AM et al. Epidemiology of candidaemia in Europe: results of 28-month European Confederation of Medical Mycology (ECMM) hospital-based surveillance study. Eur J Clin Microbiol Infect Dis. 2004;23(4):317-22.

8. Pfaller MA et al. Geographic variations in species distribution and echinocandin and azole antifungal resistance rates among Candida bloodstream infection isolates: report from the SENTRY Antimicrobial Surveillance Program (2008 to 2009). J Clin Microbiol. 2011;49(1):396-9. This are very complete studies on the epidemiology of Candida infections as well as on the antifungal resistance rates among the different Candida species. They involved more that 1,500 Candida isolates from diverse medical centers worldwide and are important to frame C. glabrata epidemiology and antifungal resistance within the globality of Candida infections. These studies also show that pre-exposure to other classes of antifungals contribute to species distribution changes, particularly the increase incidence of C. glabrata.

9. Kao AS et al. The epidemiology of candidemia in two United States cities: results of a population-based active surveillance. Clin Infect Dis. 1999;29(5):1164-70.

10. Bassetti M, et al. Epidemiology, Species Distribution, Antifungal Susceptibility, and Outcome of Candidemiaacross five sites in Italy and Spain. J Clin Microbiol. 2013.

11. Asmundsdottir LR, Erlendsdottir H, Gottfredsson M. Nationwide study of candidemia, antifungal use, and antifungal drug resistance in Iceland, 2000 to 2011. J Clin Microbiol. 2013;51(3):841-8.

12. Arendrup $\mathrm{MC}$ et al. National surveillance of fungemia in Denmark (2004 to 2009). J Clin Microbiol. 2011;49(1):325-34.

13. Falagas ME, Roussos N, Vardakas KZ. Relative frequency of albicans and the various non-albicans Candida spp among candidemia isolates from inpatients in various parts of the world: a systematic review. Int J Infect Dis. 2010;14(11):e954-66.

14. Nucci $\mathrm{M}$ et al. Epidemiology of candidemia in Latin America: a laboratory-based survey. PLoS One. 2013;8(3):e59373.

15. Colombo AL et al. Candida glabrata: an emerging pathogen in Brazilian tertiary care hospitals. Med Mycol. 2013;51(1):38-44.

16. Eggimann P, Garbino J, Pittet D. Epidemiology of Candida species infections in critically ill non-immunosuppressed patients. Lancet Infect Dis. 2003;3(11):685-702.

17. Spellberg BJ, Filler SG, Edwards Jr JE. Current treatment strategies for disseminated candidiasis. Clin Infect Dis. 2006;42(2):244-51.

18. Zaas AK, Alexander BD. Echinocandins: role in antifungal therapy, 2005. Expert Opin Pharmacother. 2005;6(10):1657-68.

19. Pfaller MA et al. Results from the ARTEMIS DISK Global Antifungal Surveillance study, 1997 to 2005: an 8.5-year analysis of susceptibilities of Candida species and other yeast species to fluconazole and voriconazole determined by CLSI standardized disk diffusion testing. J Clin Microbiol. 2007;45(6): 1735-45. 
20. Bassetti $\mathrm{M}$ et al. Epidemiology, species distribution, antifungal susceptibility, and outcome of candidemia across five sites in Italy and Spain. J Clin Microbiol. 2013;51(12):4167-72.

21. Lockhart SR et al. Species identification and antifungal susceptibility testing of Candida bloodstream isolates from populationbased surveillance studies in two U.S. cities from 2008 to 2011. J Clin Microbiol. 2012;50(11):3435-42.

22. Richet $\mathrm{H}$ et al. Candidemia in French hospitals: incidence rates and characteristics. Clin Microbiol Infect. 2002;8(7):405-12.

23. Leroy $\mathrm{O}$ et al. Epidemiology, management, and risk factors for death of invasive Candida infections in critical care: a multicenter, prospective, observational study in France (2005-2006). Crit Care Med. 2009;37(5):1612-8.

24. Ericsson $\mathrm{J}$ et al. Candidaemia in Sweden: a nationwide prospective observational survey. Clin Microbiol Infect. 2013;19(4):E218-21.

25. Orasch $\mathrm{C}$, et al. Candida Species Distribution and Antifungal Susceptibility Testing According to EUCAST and New vs. Old CLSI Clinical Breakpoints: a Six-Year Prospective Candidemia Survey from the Fungal Infection Network of Switzerland (FUNGINOS). Clin Microbiol Infect. 2013.

26. Poikonen E et al. Secular trend in candidemia and the use of fluconazole in Finland, 2004-2007. BMC Infect Dis. 2010;10:312.

27. Sandven $P$ et al. Candidemia in Norway (1991 to 2003): results from a nationwide study. J Clin Microbiol. 2006;44(6):1977-81.

28. Das I et al. Epidemiology, clinical characteristics, and outcome of candidemia: experience in a tertiary referral center in the UK. Int $\mathrm{J}$ Infect Dis. 2011;15(11):e759-63.

29. Presterl E et al. Changing pattern of candidaemia 2001-2006 and use of antifungal therapy at the University Hospital of Vienna, Austria. Clin Microbiol Infect. 2007;13(11):1072-6.

30. Sabino R et al. Epidemiology of candidemia in oncology patients: a 6-year survey in a Portuguese central hospital. Med Mycol. 2010;48(2):346-54.

31. Borst $\mathrm{A}$ et al. Rapid acquisition of stable azole resistance by Candida glabrata isolates obtained before the clinical introduction of fluconazole. Antimicrob Agents Chemother. 2005;49(2):783-7.

32. Bassetti $\mathrm{M}$ et al. Incidence of candidaemia and relationship with fluconazole use in an intensive care unit. J Antimicrob Chemother. 2009;64(3):625-9.

33. Lin MY et al. Prior antimicrobial therapy and risk for hospital-acquired Candida glabrata and Candida krusei fungemia: a case-case-control study. Antimicrob Agents Chemother. 2005;49(11):4555-60.

34. Malani A et al. Candida glabrata fungemia: experience in a tertiary care center. Clin Infect Dis. 2005;41(7):975-81.

35. Gomez-Lopez A et al. In vitro susceptibility of Cryptococcus gattii clinical isolates. Clin Microbiol Infect. 2008;14(7):727-30.

36. Hsu DI et al. A multicentre study to evaluate the impact of timing of caspofungin administration on outcomes of invasive candidiasis in non-immunocompromised adult patients. J Antimicrob Chemother. 2010;65(8):1765-70.

37. Parkins MD et al. Adequacy of empirical antifungal therapy and effect on outcome among patients with invasive Candida species infections. J Antimicrob Chemother. 2007;60(3):613-8.

38. Arendrup MC et al. EUCAST technical note on the EUCAST definitive document EDef 7.2: method for the determination of broth dilution minimum inhibitory concentrations of antifungal agents for yeasts EDef 7.2 (EUCAST-AFST). Clin Microbiol Infect. 2012;18(7):E246-7.

39. Collins CD et al. To test or not to test: a cost minimization analysis of susceptibility testing for patients with documented Candida glabrata fungemias. J Clin Microbiol. 2007;45(6):1884-8.

40. Pfaller MA, Sheehan DJ, Rex JH. Determination of fungicidal activities against yeasts and molds: lessons learned from bactericidal testing and the need for standardization. Clin Microbiol Rev. 2004;17(2):268-80.
41. Park BJ et al. Evaluation of amphotericin B interpretive breakpoints for Candida bloodstream isolates by correlation with therapeutic outcome. Antimicrob Agents Chemother. 2006;50(4):1287-92.

42. Pfaller MA. Antifungal susceptibility testing methods. Curr Drug Targets. 2005;6(8):929-43.

43. Peyron $\mathrm{F}$ et al. Improved detection of amphotericin B-resistant isolates of Candida lusitaniae by Etest. J Clin Microbiol. 2001;39(1):339-42.

44. Yang YL et al. Susceptibilities to amphotericin B and fluconazole of Candida species in TSARY 2002. Diagn Microbiol Infect Dis. 2005;51(3):179-83.

45. Canton E et al. Patterns of amphotericin B killing kinetics against seven Candida species. Antimicrob Agents Chemother. 2004;48(7): 2477-82.

46. Guinea J, et al. Molecular identification and antifungal susceptibility of yeast isolates causing fungemia Collected in a populationbased study in Spain from 2010 to 2011. Antimicrob Agents Chemother. 2013.

47. Krogh-Madsen M et al. Amphotericin B and caspofungin resistance in Candida glabrata isolates recovered from a critically ill patient. Clin Infect Dis. 2006;42(7):938-44.

48. Chen YC et al. Stable susceptibility of Candida blood isolates to fluconazole despite increasing use during the past 10 years. J Antimicrob Chemother. 2003;52(1):71-7.

49. Cuenca-Estrella $\mathrm{M}$ et al. Head-to-head comparison of the activities of currently available antifungal agents against 3,378 Spanish clinical isolates of yeasts and filamentous fungi. Antimicrob Agents Chemother. 2006;50(3):917-21.

50. Pfaller MA, Diekema DJ, G. International Fungal Surveillance Participant. Twelve years of fluconazole in clinical practice: global trends in species distribution and fluconazole susceptibility of bloodstream isolates of Candida. Clin Microbiol Infect. 2004;10 Suppl 1:11-23.

51. Subcommittee on Antifungal Susceptibility Testing of the, E.E.C.f.A.S.T. EUCAST definitive document EDef 7.1: method for the determination of broth dilution MICs of antifungal agents for fermentative yeasts. Clin Microbiol Infect. 2008;14(4):398-405.

52. Pfaller MA, Diekema DJ, Sheehan DJ. Interpretive breakpoints for fluconazole and Candida revisited: a blueprint for the future of antifungal susceptibility testing. Clin Microbiol Rev. 2006;19(2):435-47.

53. Arendrup $\mathrm{MC}$ et al. Semi-national surveillance of fungaemia in Denmark 2004-2006: increasing incidence of fungaemia and numbers of isolates with reduced azole susceptibility. Clin Microbiol Infect. 2008;14(5):487-94.

54. Pfaller MA et al. Frequency of decreased susceptibility and resistance to echinocandins among fluconazole-resistant bloodstream isolates of Candida glabrata. J Clin Microbiol. 2012;50(4):1199 203. This are very complete studies on the epidemiology of Candida infections as well as on the antifungal resistance rates among the different Candida species. They involved more that 1,500 Candida isolates from diverse medical centers worldwide and are important to frame C. glabrata epidemiology and antifungal resistance within the globality of Candida infections. These studies also show that preexposure to other classes of antifungals contribute to species distribution changes, particularly the increase incidence of C. glabrata.

55. Costa-de-Oliveira $\mathrm{S}$ et al. A first Portuguese epidemiological survey of fungaemia in a university hospital. Eur J Clin Microbiol Infect Dis. 2008;27(5):365-74.

56. Ullmann AJ et al. ESCMID* guideline for the diagnosis and management of Candida diseases 2012: adults with haematological malignancies and after haematopoietic stem cell transplantation (HCT). Clin Microbiol Infect. 2012;18 Suppl 7:53-67.

57. Hope WW et al. ESCMID* guideline for the diagnosis and management of Candida diseases 2012: prevention and management of invasive infections in neonates and children caused by Candida spp. Clin Microbiol Infect. 2012;18 Suppl 7:38-52. 
58. Sobel JD. Changing trends in the epidemiology of Candida blood stream infections: a matter for concern? Crit Care Med. 2010;38(3): 990-2.

59. Pappas PG, Mycoses Study Group. Candidemia in the intensive care unit: miles to go before we sleep. Crit Care Med. 2011;39(4): $884-5$.

60. Lortholary $\mathrm{O}$ et al. Recent exposure to caspofungin or fluconazole influences the epidemiology of candidemia: a prospective multicenter study involving 2,441 patients. Antimicrob Agents Chemother. 2011;55(2):532-8.

61. Pfaller MA et al. Echinocandin and triazole antifungal susceptibility profiles for Candida spp., Cryptococcus neoformans, and Aspergillus fumigatus: application of new CLSI clinical breakpoints and epidemiologic cutoff values to characterize resistance in the SENTRY Antimicrobial Surveillance Program (2009). Microbiol Infect Dis. 2011;69(1):45-50.

62. Mora-Duarte $\mathrm{J}$ et al. Comparison of caspofungin and amphotericin B for invasive candidiasis. N Engl J Med. 2002;347(25):2020-9.

63. Pfaller $\mathrm{M}$ et al. Use of epidemiological cutoff values to examine 9year trends in susceptibility of Candida species to anidulafungin, caspofungin, and micafungin. J Clin Microbiol. 2011;49(2):624-9.

64. Chapeland-Leclerc F et al. Acquisition of flucytosine, azole, and caspofungin resistance in Candida glabrata bloodstream isolates serially obtained from a hematopoietic stem cell transplant recipient. Antimicrob Agents Chemother. 2010;54(3):1360-2.

65. Kofteridis DP, Lewis RE, Kontoyiannis DP. Caspofungin-nonsusceptible Candida isolates in cancer patients. J Antimicrob Chemother. 2010;65(2):293-5.

66. Lewis 2nd JS et al. Rapid emergence of echinocandin resistance in Candida glabrata resulting in clinical and microbiologic failure. Antimicrob Agents Chemother. 2013;57(9):4559-61.

67. Schunemann HJ et al. Grading quality of evidence and strength of recommendations for diagnostic tests and strategies. BMJ. 2008;336(7653):1106-10.

68. Cuenca-Estrella M et al. ESCMID* guideline for the diagnosis and management of Candida diseases 2012: diagnostic procedures. Clin Microbiol Infect. 2012;18 Suppl 7:9-18. The ESCMID analyzes several diagnostic procedures and provides valuable recommendations and guideline for the use of these diagnostic procedures in several types Candida diseases, including IC.

69. Denning DW et al. British Society for Medical Mycology proposed standards of care for patients with invasive fungal infections. Lancet Infect Dis. 2003;3(4):230-40.

70. Richardson M, Ellis M. Clinical and laboratory diagnosis. Hosp Med. 2000;61(9):610-4.

71. Marchetti $\mathrm{O}$ et al. ECIL recommendations for the use of biological markers for the diagnosis of invasive fungal diseases in leukemic patients and hematopoietic SCT recipients. Bone Marrow Transplant. 2012;47(6):846-54.
72. Jensen HE, Salonen J, Ekfors TO. The use of immunohistochemistry to improve sensitivity and specificity in the diagnosis of systemic mycoses in patients with haematological malignancies. J Pathol. 1997;181(1):100-5.

73. Ene IV et al. Carbon source-induced reprogramming of the cell wall proteome and secretome modulates the adherence and drug resistance of the fungal pathogen Candida albicans. Proteomics. 2012;12(21):3164-79.

74. Yera $\mathrm{H}$ et al. Contribution of serological tests and blood culture to the early diagnosis of systemic candidiasis. Eur J Clin Microbiol Infect Dis. 2001;20(12):864-70.

75. Mikulska $\mathrm{M}$ et al. The use of mannan antigen and anti-mannan antibodies in the diagnosis of invasive candidiasis: recommendations from the Third European Conference on Infections in Leukemia. Crit Care. 2010;14(6):R222.

76. De Pauw B et al. Revised definitions of invasive fungal disease from the European Organization for Research and Treatment of Cancer/Invasive Fungal Infections Cooperative Group and the National Institute of Allergy and Infectious Diseases Mycoses Study Group (EORTC/MSG) Consensus Group. Clin Infect Dis. 2008;46(12):1813-21.

77. Posteraro B et al. Early diagnosis of candidemia in intensive care unit patients with sepsis: a prospective comparison of (1->3)-betaD-glucan assay, Candida score, and colonization index. Crit Care. 2011;15(5):R249.

78. White PL, Archer AE, Barnes RA. Comparison of non-culturebased methods for detection of systemic fungal infections, with an emphasis on invasive Candida infections. J Clin Microbiol. 2005;43(5):2181-7.

79. Stevens DA et al. Escape of Candida from caspofungin inhibition at concentrations above the MIC (paradoxical effect) accomplished by increased cell wall chitin; evidence for beta-1,6-glucan synthesis inhibition by caspofungin. Antimicrob Agents Chemother. 2006;50(9):3160-1.

80. Koo S et al. Diagnostic performance of the (1->3)-beta-D-glucan assay for invasive fungal disease. Clin Infect Dis. 2009;49(11): 1650-9.

81. Avni T, Leibovici L, Paul M. PCR diagnosis of invasive candidiasis: systematic review and meta-analysis. J Clin Microbiol. 2011;49(2):665-70.

82. Alcoba-Florez $\mathrm{J}$ et al. Phenotypic and molecular characterization of Candida nivariensis sp. nov., a possible new opportunistic fungus. J Clin Microbiol. 2005;43(8):4107-11.

83. Correia A et al. Candida bracarensis sp. nov., a novel anamorphic yeast species phenotypically similar to Candida glabrata. Int J Syst Evol Microbiol. 2006;56(Pt 1):313-7.

84. Lockhart SR et al. Identification of Candida nivariensis and Candida bracarensis in a large global collection of Candida glabrata isolates: comparison to the literature. J Clin Microbiol. 2009;47(4): 1216-7. 